\title{
Decreasing Hypoglycemia following Insulin Administration for Inpatient Hyperkalemia
}

\author{
Allen Vinh Tran, PharmD', Robert J Rushakoff, MD², Priya Prasad, PhD³, \\ Sara G Murray, MD, MAS 3 , Bradley Monash, MD³, Heidemarie Macmaster, PharmD ${ }^{4}$
}

${ }^{1}$ School of Pharmacy, University of California, San Francisco, California; ${ }^{2}$ Division of Endocrinology and Metabolism, University of California, San Francisco, California; ${ }^{3}$ Division of Hospital Medicine, University of California, San Francisco, California; ${ }^{4}$ Institute for Nursing Excellence, University of California, San Francisco, California, (currently at Lahey Health System, Burlington, Massachusetts).

A correction has been made to the Figure. A dosage was incorrect in the Orderset $1.1(1 / 1 / 16-3 / 19 / 17)$ box. The figure listed Insulin 19 Units IV $\times 1$ and should have been Insulin 10 Units IV x 1 . Below is the corrected figure.

\section{Orderset $1.1(1 / 1 / 16-3 / 19 / 17)$}

Features of the Orderset

1. Hyperkalemia Treatment Modalities

- Insulin 10 Units IV x1

- D50 25g IV x1

2. POCT Pre- and Post-Insulin Administration

\section{Orderset $1.2(3 / 20 / 17-9 / 30 / 17)$}

Features of the Orderset

1. Hyperkalemia Treatment Modalities

2. POCT Pre- and Post-Insulin Administration

3. Weight-Based Dosing for Insulin (0.1 units/kg)-NEW

4. Provider alerts to Identofy At-Risk Patients for Hypoglycemia NEW

5. Concomitant Dextrose Administration Based on Pre-Insulin Blood Glucose - NEW

-If Pre-Insulin Blood Glucose $<150 \mathrm{mg} / \mathrm{dL}$, then Add an Additional Dextrose 50\% (25g) IV Once 1-Hour Post-Insulin Admonistration.

-If Pre-Insulin BLood Glucose is $>300 \mathrm{mg} / \mathrm{dL}$, then Dextrose $50 \%$ (25g) with Insulin Administration.

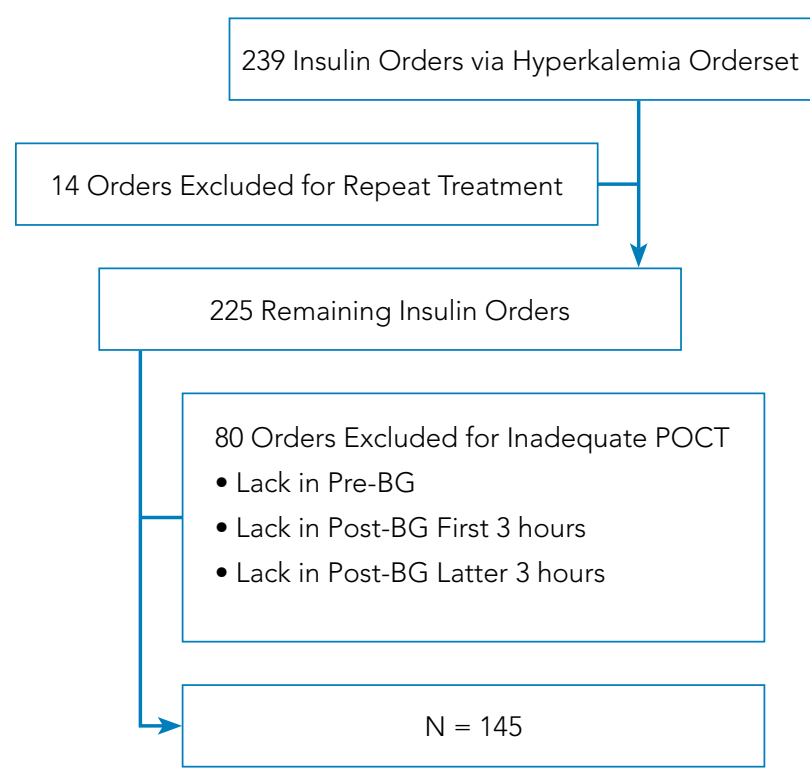

FIG. Flow Diagram Displaying the Study Cohort's Inclusion and Exclusion Criteria Abbreviation: POCT, point of care testing. 